\title{
Reconfiguración multiobjetivo en sistemas de distribución primaria de energía
}

\author{
Multiobjective reconfiguration in primary power distribution systems
}

\author{
Irina Salazar Fonseca ${ }^{1 *} \quad$ Sergio Pablo de la Fé Dotres ${ }^{1} \quad$ Gustavo Torres Guerrero $^{2}$ \\ Recibido 4 de diciembre de 2015, aceptado 22 de junio de 2016 \\ Received: December 4, 2015 Accepted: June 22, 2016
}

\begin{abstract}
RESUMEN
Este trabajo aborda el problema de la disminución de las pérdidas de potencia en las redes de distribución sin afectar la calidad del servicio eléctrico ofrecido; para lo que se propone una metodología de reconfiguración multiobjetivo de estas redes. En el planteamiento del problema se trabaja con dos funciones objetivos; el Valor Actual Neto de los Costos (VANC) y la Duración Equivalente de la Potencia Interrumpida (DEPI). Mientras que en la solución del modelo se emplea un algoritmo evolutivo elitista de ordenamiento no dominado (NSGA-II), el que se implementa con ayuda del MATLAB, determinando un conjunto de soluciones factibles. La metodología propuesta es implementada en la reconfiguración de los circuitos Y-410, Y-283 y Y-285 de la ciudad de Camagüey, Cuba, donde se logran reducir las pérdidas de potencia de la red analizada en $51,7 \mathrm{~kW}$ y mejorar la calidad del servicio que se brinda, disminuyendo a 0,3966 horas/año las horas equivalentes en que falla el servicio eléctrico a cada kVA instalado en los clientes.
\end{abstract}

Palabras clave: NSGA-II, pérdidas de potencia, reconfiguración multiobjetivo de redes de distribución.

\begin{abstract}
This paper presents the problem of power loss reduction in the distribution power systems without affecting the supplied electrical service quality. An approach of this problem using a methodology for the multi-objective reconfiguration is proposed. In the statement of the problem two objective functions are used: The Net Actual Value of Costs (VANC) and the Equivalent Duration of Interrupted Power (DEPI). While in the model's solution a Non-dominated Sorting Genetic Algorithm (NSGA-II) is used, which is an evolutionary algorithm implemented in MATLAB from where a feasible set of solutions is obtained. The methodology proposed is used in the reconfiguration of circuits Y-410, Y-283 and Y-285 in the Camagüey city, Cuba, where the power losses were reduced in $51.7 \mathrm{~kW}$, improving in that way the quality of the service supplied, diminishing to 0.3966 hours/year the equivalent hours in which the electric service fails for each $\mathrm{kVA}$ installed in the clients.
\end{abstract}

Keywords: NSGA-II, power losses, multi-objective reconfiguration of distribution power systems.

1 Departamento de Ingeniería Eléctrica. Universidad de Oriente. Ave. De las Américas s/n. Santiago de Cuba, Cuba. CP 90100. E-mail: isalazar@uo.edu.cu; sergiof@uo.edu.cu

2 Departamento de Matemáticas. Universidad de Oriente. Ave. De las Américas s/n. Santiago de Cuba, Cuba. CP 90100. E-mail: gtorres@uo.edu.cu

* Autor de correspondencia 


\section{INTRODUCCIÓN}

El objetivo de las redes de distribución es mantener en los terminales de los consumidores de energía eléctrica un nivel de tensión adecuado con una cierta confiabilidad. El crecimiento de la demanda de energía eléctrica y el envejecimiento de los componentes de la red provocan un incremento de las pérdidas de potencia activa con el consecuente incremento de los costos de explotación y de las interrupciones del servicio eléctrico. Cuando esto sucede, se justifica, tanto desde el punto de vista técnico como económico, mejorar la red de distribución.

La reconfiguración de las redes de distribución es una mejora que emplea las características de la topología de dichas redes, para modificarla, y así reducir las pérdidas eléctricas y reducir el número de clientes afectados por una falla. Las redes de distribución se operan radiales y se construyen malladas, por lo tanto, es posible intercambiar carga entre un alimentador y otro, hasta obtener un punto de operación adecuado [1].

La importancia de emplear dos o más objetivos en la solución del problema radica en una mejor aproximación del modelo matemático empleado para describir los sistemas reales [2].
En la literatura especializada se encuentran algunos trabajos que procuran diversos objetivos. En [3] se presenta un modelo que considera como funcionesobjetivo los costos y confiabilidad del sistema, y en la solución usan un algoritmo de búsqueda tabú. En [4] emplean un modelo matemático no lineal entero mixto que minimiza dos funciones-objetivo: económica y confiabilidad, y en la solución se valen de un algoritmo genético especializado. En $[2,5]$ se emplea un algoritmo elitista de ordenamiento no dominado (NSGA-II), en la solución del problema multiobjetivo, donde el modelo matemático empleado es no lineal entero mixto.

Por lo que en el presente artículo en la solución del problema se emplea un algoritmo evolutivo elitista de ordenamiento no dominado (NSGA-II), elaborado por [6].

\section{ANÁLISIS TEÓRICO}

La reconfiguración de alimentadores primarios es la alteración de la estructura topológica a partir de la apertura o el cierre de seccionalizadores que son interruptores normalmente cerrados (NC) y los enlaces que son interruptores normalmente abiertos (NA) [7], Figura 1. A su vez, es el proceso por medio del que se transfieren cargas de un alimentador a otro, teniendo en cuenta las restricciones de los

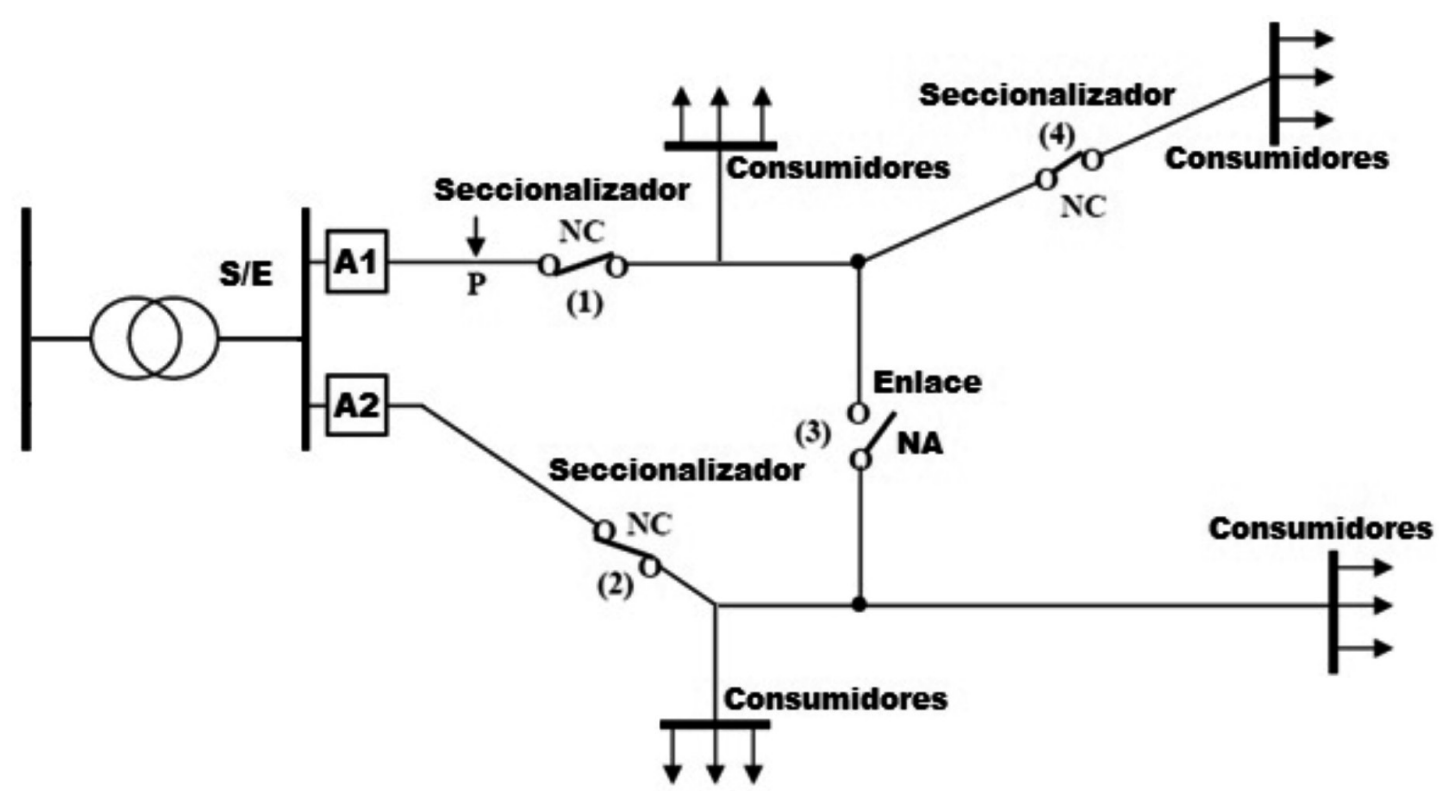

Figura 1. Red radial con una subestación y dos alimentadores primarios. 
parámetros de operación de la red y la topología de los circuitos.

La topología que se seleccione debe cumplir con las restricciones mínimas que son:

- Conservar la configuración radial de los alimentadores.

- Todas las cargas deben ser servidas, en el caso de que se realice la reconfiguración para disminuir pérdidas. Si el objetivo es mejorar la confiabilidad del sistema entonces se trata que la mayor cantidad de clientes sean alimentados.

- Los límites térmicos de líneas, transformadores y otros equipos no deben ser excedidos.

- Los niveles de tensión tienen que quedar dentro de los límites establecidos.

Existen números, métodos para desarrollar la reconfiguración, que van desde los más elementales y de fácil implementación hasta los más complejos que pueden obtener resultados precisos, que se supone va a ofrecer una buena solución (no necesariamente la óptima). Estos métodos podemos agruparlos en dos grupos: los métodos basados en conocimientos y métodos basados en técnicas evolutivas.

1. Métodos basados en conocimientos:

- Técnicas heurísticas.

- Programación lineal.

- Redes neuronales.

- Sistemas expertos.

- Lógica difusa.

2. Métodos basados en técnicas evolutivas:

- Técnicas de enfriamiento simulado.

- Técnicas de algoritmos genéticos.

Hoy en día existe una tendencia a la optimización de los problemas bajo una mirada más amplia por medio de un tratamiento multiobjetivo. Por esta razón, en los últimos años ha existido un creciente desarrollo de nuevas técnicas de reconfiguración fundamentadas principalmente en métodos basados en técnicas evolutivas.

\section{Formulación matemática del problema}

Teniendo en cuenta los aspectos expuestos, el presente trabajo formula el problema de la reconfiguración de las redes de distribución, como un problema multiobjetivo que persigue la localización de los interruptores de enlace necesarios para minimizar:
1. El Costo del Ciclo de Vida (VANC).

2. Duración Equivalente de la Potencia Interrumpida (DEPI).

Sujeto a restricciones técnicas:

- Caídas de tensión permitidas.

- Capacidad máxima admisibles de subestaciones y alimentadores.

- Mantener la configuración radial del esquema.

\section{Función-objetivo (VANC):}

En este caso, los indicadores a tener en cuenta están relacionados con los gastos asociados a la operación de las redes de distribución, ellos son:

- Gastos de depreciación $(\mathrm{Ca})$.

- Gastos de mantenimiento ( $\left.\mathrm{C}_{\mathrm{MTTO}}\right)$.

- Gastos de pérdidas de energía activa $\left(\mathrm{C}_{\Delta \mathrm{E}}\right)$.

$$
V A N C=K_{t}+\sum_{j=1}^{n} \frac{C_{\Delta E}+C a+C_{M T T O}}{(1+T D)^{j}}
$$

donde $\mathrm{K}_{\mathrm{t}}$ es la inversión o capital inicial, (incluye equipos, construcción y montaje y otros) en $\$$; TD es la tasa de descuento establecida (la Unión Nacional Eléctrica establece para estos análisis valores de 10, 12 y $15 \%$ ) y n son los años de explotación.

$$
C a=\phi \cdot K_{0}
$$

$\mathrm{K}_{0}$ es la inversión inicial de los equipos tecnológicos en $\$$ y $\phi$ es el coeficiente anual de descuento por depreciación, según la Resolución 399/2003 del Ministerio de Finanzas y Precios, este coeficiente toma el valor de un $10 \%$ anual para equipos tecnológicos.

$$
C_{\text {MTTO }}=\psi \cdot K_{t}
$$

$\psi$ es el coeficiente anual de descuento por mantenimiento, este coeficiente toma el valor de un $2 \%$ anual.

$$
C_{\Delta E}=\left(\frac{C C \cdot C E}{(1-F D P)}\right) \cdot \Delta E_{n}
$$

CC es el costo del combustible en \$/Ton; CE es el consumo específico en Ton/MW-h y FPD es el factor de pérdidas en distribución, dato suministrado por la Empresa Eléctrica de Santiago de Cuba.

$$
\Delta E_{n}=\Delta E_{0} \cdot\left(1+\alpha_{i}\right)^{2 \cdot(n-1)}
$$


donde $\Delta \mathrm{E}_{\mathrm{n}}$ son las pérdidas de energía en los años de análisis, en $\mathrm{kW}-\mathrm{h} ; \Delta \mathrm{E}_{0}$ son las pérdidas de energía en año 0 , en $\mathrm{kW}-\mathrm{h} ; \alpha_{i}$ es el porcentaje de crecimiento anual y $\mathrm{n}$ es el número de años considerados, en el caso de las pérdidas el año 0 es el primer año de estudio.

\section{Función-objetivo (DEPI):}

Este indicador tiene la ventaja principal de permitir trabajar directamente con los datos que existen en las redes de distribución cubanas, los kVA instalados en cada banco de transformadores.

$$
D E P I=\frac{\sum_{i=1}^{n}\left(\lambda_{i} \cdot S_{i} \cdot T M R S_{i}\right)}{S_{t}}
$$

donde $\mathrm{S}_{\mathrm{i}}$ es la potencia instalada en kVA del tramo; $\mathrm{S}_{\mathrm{t}}$ es la potencia instalada en kVA total; $\mathrm{TMRS}_{\mathrm{i}}$ es el tiempo medio de restauración por tramos en h y $\lambda_{\mathrm{j}}$ es la tasa de falla del tramo en veces/año.

$$
\lambda_{i}=\frac{m}{L \cdot T} \cdot l
$$

$m$ es la cantidad de fallas; $L$ es la longitud total de las líneas expuestas a la falla en $\mathrm{km}$; T es el período de estudio en años y 1 es la longitud de la línea de interés en $\mathrm{km}$.

Como consideraciones del problema aproximado (condiciones iniciales) se plantean las siguientes:

- Todas las líneas poseen interruptores que pueden cambiar su estado de abierto a cerrado, excepto el primer tramo de los alimentadores donde se encuentran instalados los interruptores generales.

- Los enlaces se realizarán en los troncos de los circuitos y en aquellos ramales que sean trifásicos.

- Se definirán las mallas elementales del sistema, como aquella malla que puede ser formada en el sistema y que no contiene a otras mallas.

\section{Restricciones:}

Caídas de tensión.

$$
\% \Delta V_{i} \leq \% \Delta V_{n}
$$

donde $\% \Delta \mathrm{V}_{\mathrm{i}}$ es el porcentaje calculado en el nodo i-ésimo del sistema de distribución; $\% \Delta \mathrm{V}_{\mathrm{n}}$ es el porcentaje máximo permitido, sobre la tensión nominal. Según NC-365:2004, la tensión máxima no difiere en más $5 \%$ y la tensión mínima en menos $10 \%$ de la tensión nominal del sistema.

Capacidad máxima admisibles de subestaciones $y$ alimentadores.

La capacidad máxima admisibles de subestaciones y alimentadores se establece de acuerdo a las restricciones siguientes:

$$
\begin{gathered}
I_{\text {máx, } i j} \leq I_{l t, i j} \\
S_{\text {máx }} \leq S_{n}
\end{gathered}
$$

donde $I_{m a ́ x, i j}$ es la corriente máxima que circula por el tramo de línea $i, j ; S_{m a ́ x}$ es la potencia aparente máxima demandada por el circuito y $S_{n}$ es la capacidad nominal de la subestación

\section{Mantener la configuración radial del esquema.}

El modelo planteado en este trabajo permite la selección de topologías radiales, donde no pueden existir caminos en la topología que permitan la formación de mallas o lazos ni nodos sin alimentación.

- Para detectar nodos sin alimentación se explora la matriz de incidencia A en busca de alguna columna donde todos los elementos sean ceros, en ese caso existe un nodo sin alimentación y es descartada esa configuración.

- Para detectar si una configuración es radial, se construye la matriz de adyacencia nodo-nodo, luego se explora en busca de caminos cerrados, de encontrarse se descarta la configuración.

La técnica de solución empleada para resolver el problema de la reconfiguración multiobjetivo de las redes de distribución es el algoritmo NSGA-II, elaborado por [6]. Esta metodología presenta dos características importantes: garantiza la diversidad durante el proceso de solución y además se caracteriza por ser elitista, es decir, considera las mejores soluciones encontradas durante el proceso de búsqueda.

Las principales etapas del NSGA-II se describen a continuación:

1. Generar una población inicial Pt de tamaño $\mathrm{N}$ de forma aleatoria.

2. Se identifican los frentes de dominancia de la forma siguiente, por cada solución, se calculan dos 
valores ni, el número de soluciones que dominan a la solución i, y Si, un conjunto de soluciones en las que la solución i domina. Posteriormente se identifican todos los puntos que tienen $\mathrm{ni}=0$ y se almacenan en una lista F1 (frontera actual). Luego, para cada solución en la frontera actual se visita cada miembro (j) en su conjunto Si y reduce su nj uno a uno. De esta forma, si para cualquier miembro de $\mathrm{j}$ el valor se vuelve cero, se pone en una lista separada $\mathrm{H}$. Cuando todos los miembros de la frontera actual han sido chequeados, se declara a los miembros de la lista $\mathrm{F}_{1}$ como miembros de la primera frontera. Luego se continúa el proceso usando la nueva frontera $\mathrm{H}$ como la frontera actual.

3. Para mantener la diversidad en la frontera de Pareto, se calculan las distancias de apilamiento en cada frente. Para esto se debe calcular el perímetro del cuboide formado por las soluciones vecinas que poseen el mismo rango de dominancia que la alternativa i, lo que se describe por medio de la ecuación (11):

$$
d_{\mathrm{i}}=\sum_{\mathrm{m}=1}^{\mathrm{M}}\left|\frac{\mathrm{fm}^{\mathrm{I}_{\mathrm{i}+1}^{\mathrm{m}}}-\mathrm{fm}^{\mathrm{I}_{\mathrm{i}-1}^{\mathrm{m}}}}{f m^{\max }-f m^{\min }}\right|
$$

$\mathrm{I}^{\mathrm{m}}$ es un vector que indica la alternativa de solución vecina a la alternativa i. $\mathrm{f}_{\mathrm{m}}{ }^{\max } \mathrm{y}_{\mathrm{m}}{ }^{\text {min }}$ son los valores máximos y mínimos sobre todo el espacio de soluciones de la función objetivo $\mathrm{m}$, y $\mathrm{M}$ es el número de funciones objetivos optimizadas.

4. Se escogen los padres usando selección por torneo, con el cruzamiento y la mutación se genera una población descendiente $\left(\mathrm{Q}_{\mathrm{t}}\right)$ del mismo tamaño $\mathrm{P}_{\mathrm{t}}$. Posteriormente se realiza una combinación entre la población de padres y descendientes para obtener una población $\mathrm{R}_{\mathrm{t}} \mathrm{de}$ tamaño $2 * \mathrm{~N}$. La población $\mathrm{R}_{\mathrm{t}}$ es organizada en frentes de Pareto mediante el uso del concepto de dominancia de Pareto $\left(\mathrm{F}_{1}, \mathrm{~F}_{2}, \ldots, \mathrm{F}_{\mathrm{n}}\right)$.

5. Una vez realizado el proceso de ordenamiento no dominado, la nueva población $\mathrm{P}_{\mathrm{t}+1}$ es generada a partir de las configuraciones de los frentes no dominados, empezando por el mejor frente $\left(F_{1}\right)$, continúa con las soluciones del segundo frente y así sucesivamente. Como la población Rt es de tamaño $2 * \mathrm{~N}$ mientras que la población $\mathrm{P}_{\mathrm{t}+1}$ es de tamaño $\mathrm{N}$, entonces no todos los frentes podrán formar parte de la nueva población, por lo que las soluciones del último frente con menor distancia de apilamiento deben ser eliminadas.

6. Si se cumple el criterio de convergencia, se finaliza el proceso. De lo contrario retornar al paso 4.

\section{Codificación del problema}

La codificación del problema de la reconfiguración de redes de distribución se describe por medio de un vector de números reales, que identifican aquellos elementos de maniobra que se encuentran presentes en líneas asociadas a las mallas fundamentales de la red de distribución.

Bajo esta estrategia se define una malla fundamental, como aquella malla que puede ser formada en el sistema y que no contiene a otras mallas, Figura 2.

La representación genética de la cadena de cromosomas que representa una topología radial en la Figura 2 es la siguiente:

[35 363738 39]

Lo que significa que los interruptores L35, L36, L37, L38 y L39 están abiertos.

\section{Métrica para determinar la mejor solución del} frente de Pareto

Luego de obtener el conjunto de soluciones óptimas (frente de Pareto) entregadas por el algoritmo, se

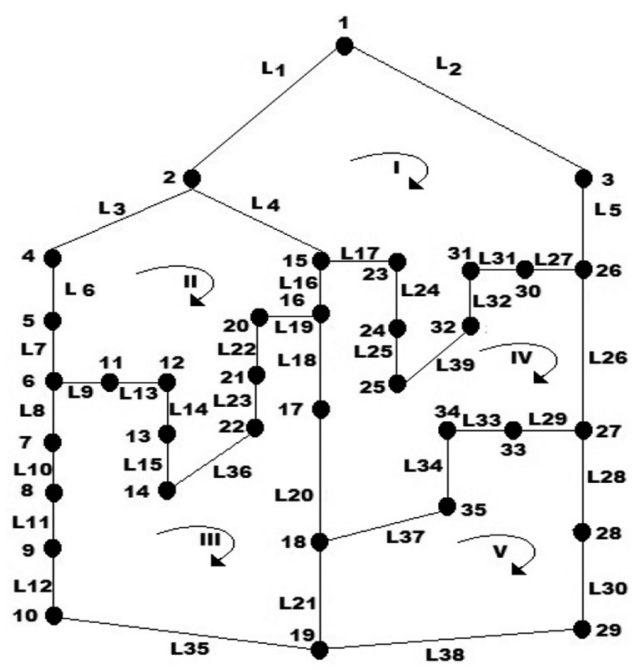

Figura 2. Ilustración de las mallas fundamentales. 
emplea el criterio Max-min expuesto en [8] para elegir la mejor solución.

Este criterio consiste en utilizar el grado de satisfacción de cada objetivo, que se puede calcular teniendo en cuenta el valor mínimo y máximo de ese objetivo. El valor mínimo tendrá un grado de satisfacción unidad y el valor máximo tendrá un grado de satisfacción cero. El valor de cada objetivo obtenido en cada solución es ordenado entre el grado máximo posible de satisfacción y el mínimo correspondiente. El criterio de decisión se basa en maximizar el grado mínimo de satisfacción de cada uno de los objetivos.

A los pares de valores normalizados se les aplica el criterio Max-min (ecuación (12)) y se obtiene la solución elegida.

$$
\max \left\{\min \left[\left(\frac{F O_{1-\max }-F O_{1-i}}{F O_{1-\max }-F O_{1-\min }}, \frac{F O_{2-\max }-F O_{2-i}}{F O_{2-\max }-F O_{2-\min }}\right)\right]\right\}
$$

Donde, $\mathrm{FO}_{1-\max }$ es el valor máximo obtenido de la función del VANC para el frente de Pareto; $\mathrm{FO}_{1-\min }$ es el valor mínimo obtenido de la función del VANC para el frente de Pareto; $\mathrm{FO}_{2 \text {-max }}$ es el valor máximo obtenido de la función DEPI para el frente de Pareto; $\mathrm{FO}_{2 \text {-min }}$ es el valor mínimo obtenido de la función DEPI para el frente de Pareto; $\mathrm{FO}_{1-\mathrm{i}}$ es el valor de la función del VAN de la solución i del frente de Pareto y $\mathrm{FO}_{2-\mathrm{i}}$ es el valor de la función de DEPI de la solución i del frente de Pareto.

\section{Metodología para la reconfiguración multiobjetivo de las redes de distribución}

Paso 1: Recopilar la información elemental para cada circuito de distribución:

- El plano del circuito de la red de distribución primaria de Media Tensión, con sus correspondientes puntos de enlaces con otros circuitos, la subestación a la que pertenece y los elementos de maniobra. El calibre de los conductores y las distancias entre los nodos.

- Potencia instalada en cada sección o tramo del circuito (kVA).

- Potencia total instalada en el circuito (kVA)

- Medición en el inicio del alimentador por el período de 24 horas.
- Cantidad de transformadores instalados en el circuito, la cantidad de clientes asociados a cada transformador, el tipo de cliente (residencial, industrial o mixto).

- Mediciones de la potencia activa y reactiva en cada transformador por un (generalmente de 24 horas) o la energía facturada en el mes.

- Número de interrupciones agrupadas según la sección del circuito afectada durante un período (generalmente 1 año).

Paso 2: Con las mediciones en el inicio del circuito se determinan el factor de potencia y el factor de carga $(\mathrm{Fc})$ del circuito:

$$
F c=\frac{\frac{1}{T} \sum_{1}^{T} P_{i}(t)}{P_{\text {máx }}}=\frac{P_{\text {med }}}{P_{\text {máx }}}
$$

Paso 3: Se seleccionan los puntos de enlaces entre los circuitos para obtener las mallas elementales.

- Además de los puntos de enlaces existentes entre los circuitos en estudios. Se pueden escoger como enlaces nodos que pertenezcan al tronco del circuito o a los ramales trifásicos, que se encuentren lo más cerca posibles unos de otros. El número de enlaces dependerá del tamaño del sistema, según [9] en sistemas grandes el número puede llegar a ser hasta 10 .

Paso 4: Concentración de la carga de los ramales monofásicos y bifásicos en el tronco de los circuitos en estudio.

Paso 5: Se realiza el diagrama general de los circuitos a reconfigurar.

Paso 6: Enumerar las mallas elementales del sistema en estudio de forma consecutiva comenzando por la malla I.

Paso 7: Se ejecuta el algoritmo NSGA-II propuesto. Paso 8: Con los resultados obtenidos del paso anterior se selecciona la mejor solución empleando la ecuación (13).

\section{RESULTADOS}

Aplicación de la metodología para la reconfiguración multiobjetivo en los circuitos en estudio Se verificará la metodología propuesta, en la reconfiguración de los circuitos Y-410, Y-283 y Y-285 de la ciudad de Camagüey. La información necesaria para el estudio fue proporcionada por la Empresa Eléctrica Provincial Camagüey. En la Tabla 1 aparecen los datos necesarios. 
El calibre de los conductores para los tres circuitos en estudio, es el siguiente:

- Tronco: Aluminio 150, con una corriente de límite térmico de $445 \mathrm{~A}$ y una impedancia específica de $(0,21+\mathrm{j} 0,40) \Omega / \mathrm{km}$.

- Ramales: Aluminio 70, con una corriente de límite térmico de $265 \mathrm{~A}$ y una impedancia específica de $(0,33+\mathrm{j} 0,43) \Omega / \mathrm{km}$.

Tabla 1. Resumen de los datos brindados por la Empresa Eléctrica de Camagüey.

\begin{tabular}{|c|c|c|c|c|c|c|}
\hline 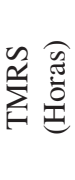 & 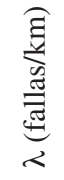 & 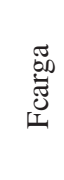 & $\overline{0}$ & 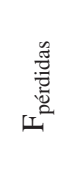 & 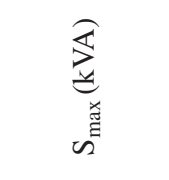 & 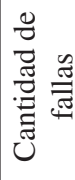 \\
\hline \multicolumn{7}{|c|}{ Y-410 } \\
\hline 3,35 & 2,05 & 0,71 & 0,95 & 0,65 & $1.660+j 520$ & 17 \\
\hline \multicolumn{7}{|c|}{$\mathrm{Y}-283$} \\
\hline 3,28 & 1,56 & 0,46 & 0,99 & 0,39 & $790+\mathrm{j} 100$ & 5 \\
\hline \multicolumn{7}{|c|}{ Y-285 } \\
\hline 4,22 & 0,98 & 0,48 & 0,98 & 0,40 & $2.020+j 460$ & 3 \\
\hline
\end{tabular}

Una vez seleccionados los enlaces y concentrada la carga se elabora el diagrama general y se enumeran las mallas elementales, como se muestra la Figura 3.

Como se puede apreciar en la Figura 3, la red a reconfigurar tiene 66 nodos, 69 ramas y cuatro mallas elementales.
Con ayuda del MATLAB, versión 7.10.0 (R2010a), se programó el algoritmo NSGA-II, los parámetros del algoritmo que presentan el mejor comportamiento se determinaron con pruebas de tanteo. Los índices de cruce y de mutación se establecieron en valores respectivos de 20 y 0,05 . Se realizaron 50 generaciones del algoritmo NSGA-II con una población de 50 individuos.

En la Tabla 2 se muestran los valores de los principales parámetros utilizados en los algoritmos evolutivos, empleados en este trabajo.

Tabla 2. Parámetros principales del algoritmo NSGA-II.

\begin{tabular}{|l|c|}
\hline Tamaño de la población & 50 \\
\hline Índice de distribución de cruce $\left(\eta_{\mathrm{c}}\right)$ & 20 \\
\hline Método de selección & Torneo Binario \\
\hline Número de generaciones & 50 \\
\hline Método de mutación & Polinomial \\
\hline Índice de distribución de mutación $\left(\eta_{\mathrm{m}}\right)$ & 0,05 \\
\hline
\end{tabular}

Ejecutado el programa con los parámetros que aparecen en la Tabla 2, se obtiene el frente de Pareto que aparece en la Figura 4.

Los datos para evaluar la primera función objetivo, fueron proporcionados por la Empresa Eléctrica Provincial de Camagüey.

$\mathrm{Kt}=75332,3 \$$ por concepto de instalación de interruptores automáticos.

$\mathrm{CC}=343,14 \$$ Ton

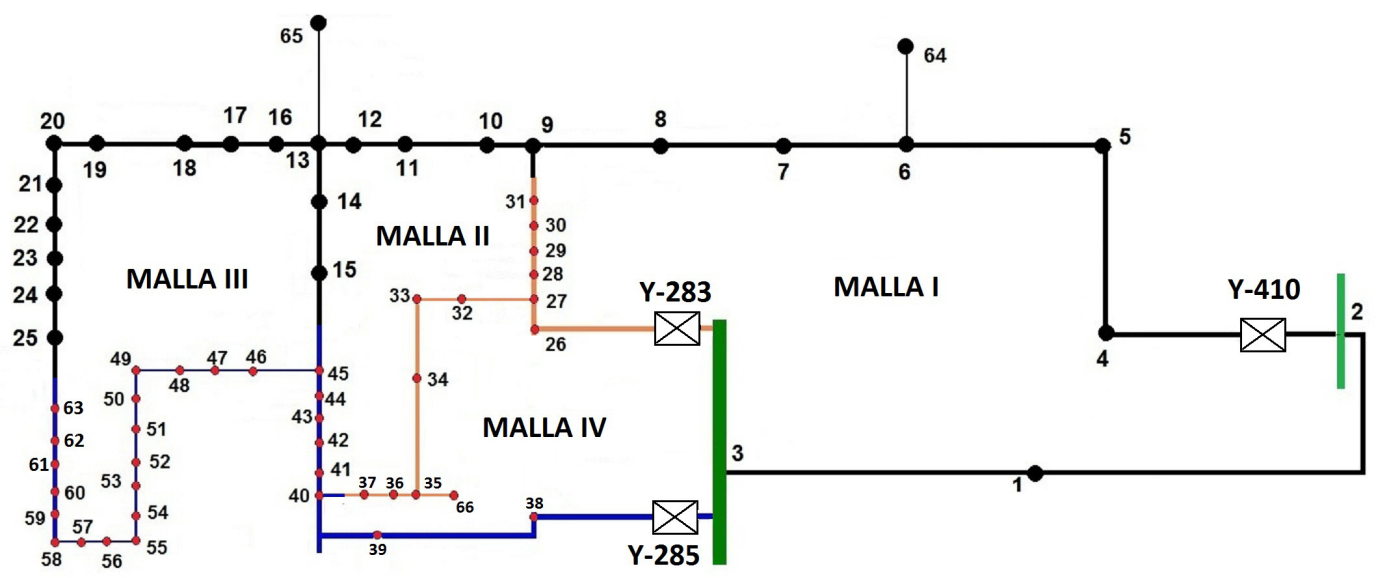

Figura 3. Esquema general para la reconfiguración. 
$\mathrm{CE}=0,27 \mathrm{Ton} / \mathrm{MW}-\mathrm{h}$

$\mathrm{FPD}=17 \%$

En la Figura 4 se ilustra el conjunto de soluciones óptimas del frente de Pareto, donde se observa que están distribuidas adecuadamente en el espacio de solución de las funciones objetivo.

Luego de obtener el frente de soluciones de Pareto, se emplea el criterio de selección de Max-min, ecuación (12), para seleccionar la mejor solución aportada por esta métrica. La configuración seleccionada por el criterio presenta la siguiente topología [18 6437 9], con un VANC igual a M\$ 567,316 y un DEPI de 0,3966 horas equivalentes por cada kVA instalado.

Esta topología indica que las líneas que se encuentran entre los nodos que se mencionarán a continuación están abiertas; [16-17, 15-45, 36-37, 7-8]. En

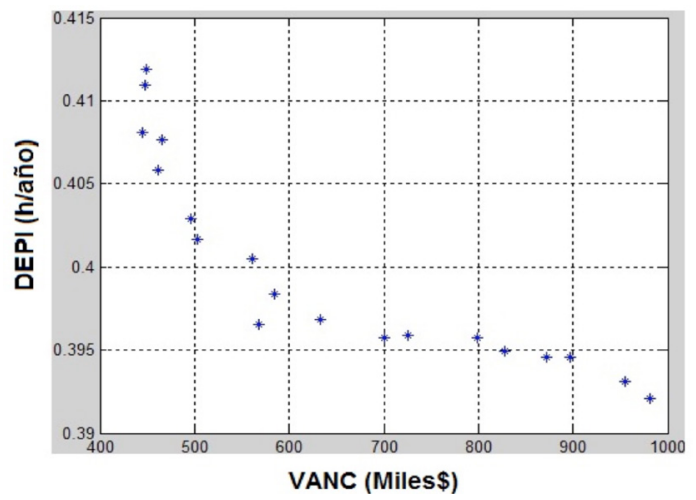

Figura 4. Conjunto de soluciones óptimas de Pareto. la Figura 5 se ilustra la topología del sistema correspondiente a la solución escogida.

Además, esta reconfiguración propuesta logra reducir las pérdidas de potencia de la red analizada en $51,7 \mathrm{~kW}$.

\section{CONCLUSIONES}

La utilización de los algoritmos evolutivos como técnica de solución del problema de optimización multiobjetivo, permite analizar simultáneamente dos objetivos con diferentes soluciones óptimas individuales, donde un objetivo generalmente se encuentra en conflicto con el otro.

La metodología propuesta tiene como ventaja, que permite la reconfiguración multiobjetivo de las redes de distribución, brindando un conjunto de soluciones de buena calidad entre los diferentes objetivos considerados. Además de ser ajustable según la experiencia acumulada por el personal de operación de las redes, las condiciones iniciales del estudio y características particulares de la región donde se encuentra ubicada la red eléctrica de distribución primaria. $\mathrm{La}$ misma permite reconfigurar circuitos de distribución minimizando las pérdidas y el DEPI simultáneamente. Obteniendo configuraciones que cumplen con todas las restricciones técnicas del problema.

La configuración escogida para los circuitos Y-410, Y-283 y Y-285, una vez aplicada la metodología propuesta, demuestra una mejora en la eficiencia y confiabilidad del servicio eléctrico que brindan. Al

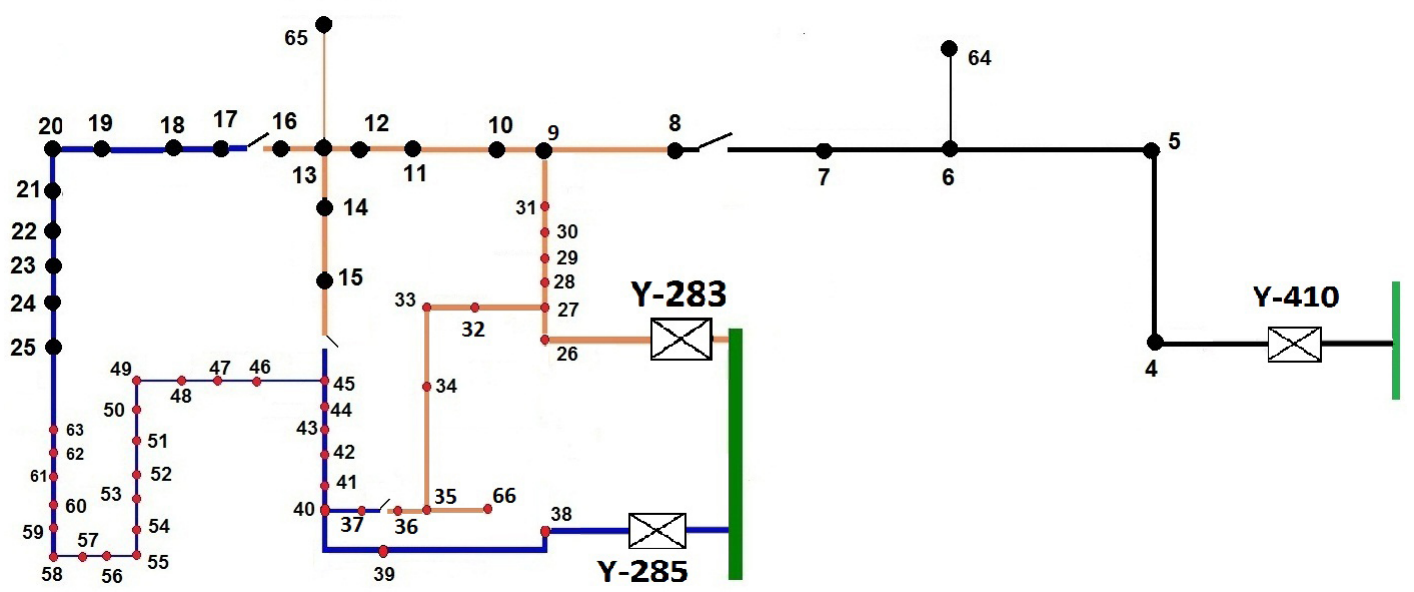

Figura 5. Esquema de la solución seleccionada. 
disminuir en \$ 35.876 al año los gastos por pérdidas de energía y 0,3583 horas equivalentes en que falla el servicio eléctrico a cada kVA instalado.

\section{REFERENCIAS}

[1] J.A. González e I. Lisan. "Algoritmo heurístico para la reconfiguración de sistemas de distribución mediante intercambio de ramas". Ingeniería Energética. Vol. 33 No 3, pp. 196204. Septiembre 2012. ISSN: 1815-5901. URL: http://scielo.sld.cu/pdf/rie/v33n3/rie03312.pdf

[2] L. López y R.A. Hincapié. "Planteamiento multiobjetivo de sistemas de distribución usando un algoritmo evolutivo NSGA-II". Revista EIA. No 15, pp. 141-151. Julio 2011. ISSN: 1794-1237. URL: http://www.scielo. org.co/ pdf/eia/n15/n15a12.pdf

[3] I. Ramírez and J. Domínguez. "New multiobjective tabu search algorithm for fuzzy optimal planning of power distribution systems". IEEE Transactions on Power Systems. Vol. 21 N$^{\circ}$ 1, pp. 224-233. February, 2006.

[4] E. Carrano, L. Soares, R. Takahashi, R. Saldanha and O. Neto. "Electric distribution network multiobjective design using a problem-specific genetic algorithm". IEEE Transactions on Power Delivery. Vol. 21 No 2, pp. 995-1005. April, 2006.
[5] R.I. Bolaños y R.A. Hincapié. "Reubicación óptima de transformadores de distribución usando el algoritmo multiobjetivo de optimización NSGA II". Ingeniería y Ciencia. Vol. $10 \mathrm{~N}^{\circ}$ 19, pp. 77-97. Junio 2014. ISSN: 1794-9165. URL: http://www.scielo.org.co/ pdf/ince/v10n19/v10n19a05.pdf

[6] D. Kalyanmoy, P. Amrit, A. Samir and T. Meyarivan. "A Fast and Elitist Multiobjective Genetic Algorithm: NSGA-II". IEEE Transactions on Evolutionary Computation. Vol. $6 \mathrm{~N}^{\circ}$ 2, pp. 182-197. 2002.

[7] B. Enacheanu and B. Raison. "Radial network reconfiguration using genetic algorithm based on the matroid theory". IEEE Transactions on Power Systems. Vol. 23 N$^{\circ}$ 1, pp. 186194. February, 2008.

[8] I. Ramírez and J. Domínguez. "Possibilistic model based on fuzzy sets for the multiobjective optimal planning of electric power distribution networks". IEEE Transactions on Power Systems. Vol. 19 N$^{\circ}$ 4, pp. 1801-1809. 2004.

[9] O. Álvarez, M.E. Bravo y Y. Brito. "Automatización de la red de $34,5 \mathrm{kV}$ en la provincia de Villa Clara". Ingeniería Energética. Vol. 33 No 3, pp. 205-216. Septiembre 2012. ISSN 1815-5901. URL: http://scielo.sld.cu/pdf/rie/v33n3/ rie04312.pdf 